University of New Hampshire

University of New Hampshire Scholars' Repository

Physics Scholarship

Physics

6-1998

\title{
The discovery of trapped energetic electrons in the outer cusp
}

\author{
R. B. Sheldon
}

Harlan E. Spence

Boston University, harlan.spence@unh.edu

J. D. Sullivan

T. A, Fritz

Jiasheng Chen

Follow this and additional works at: https://scholars.unh.edu/physics_facpub

Part of the Physics Commons

\section{Recommended Citation}

Sheldon, R. B.; Spence, Harlan E.; Sullivan, J. D.; Fritz, T. A,; and Chen, Jiasheng, "The discovery of trapped energetic electrons in the outer cusp" (1998). Geophysical Research Letters. 292.

https://scholars.unh.edu/physics_facpub/292

This Article is brought to you for free and open access by the Physics at University of New Hampshire Scholars' Repository. It has been accepted for inclusion in Physics Scholarship by an authorized administrator of University of New Hampshire Scholars' Repository. For more information, please contact Scholarly.Communication@unh.edu. 


\title{
The discovery of trapped energetic electrons in the outer cusp
}

\author{
R. B. Sheldon, H. E. Spence, J. D. Sullivan, T. A. Fritz and Jiasheng Chen \\ Boston University Center for Space Physics, Boston, Massachusetts
}

\begin{abstract}
We report on the POLAR/ CEPPAD discovery of a trapped, $60^{\circ}<\theta<120^{\circ}$ pitch angle electron population in the outer cusp (7-9+ Re), whose energetic electron component extends from below $30 \mathrm{keV}$ to $\sim 2 \mathrm{MeV}$. Because the time variability in the outer cusp precludes mapping with POLAR, we have carried out test particle simulations using the Tsyganenko 1996 model (T96) to demonstrate the trapping of these energy electrons in the outer cusp region and the resonant frequencies of its trapped motion. We discuss the boundaries and regions of the cusp trap and show that it is analogous to the dipole trap. We show that the phase space densities observed there are equal or greater than the phase space densities observed in the radiation belts at constant magnetic moment, thus allowing the possibility of diffusive filling of the radiation belts from the cusp.
\end{abstract}

\section{Introduction}

The Earth's magnetosphere has the important topological property of a magnetic bottle, field lines that have stronger magnetic field strength at the ends than in the middle, thereby permitting the trapping of plasma via the mirror force and the azimuthal symmetry of the field. This property was in retrospect identified by Störmer in 1911, but required the discovery of the radiation belts by Van Allen in 1957 before its broad application to the field of magnetospheric physics. However, what neither Störmer theory nor previous observations have predicted, is the presence of a second, quasipermanently trapped energetic particle population in the region of the Earth's outer cusp, 8-11 $R_{e}$. We call this "quasi-trapping" because the time variations in the field prevent an infinite trapping time, though in a stationary magnetic field geometry, the trapping time is infinite.

\section{Data Analysis}

POLAR is in a $2 \times 9$ Re orbit that on October 14, 1996, passed through the nominal outer cusp before traversing the radiation belts. The outer cusp is defined to be a region inside and adjacent to the mag-

Copyright 1998 by the American Geophysical Union.

Paper number $98 \mathrm{GL} 01399$.

0094-8534/98/98GL-01399\$05.00 netopause (8-10 Re), with noticeably reduced magnetic field strengths, having broadband electrostatic and magnetic wave power, and generally within some radial distance (2-3 $\mathrm{Re}$ ) of the topological minimum $B$ point. We do not define the outer cusp with respect to a particle population for the same reason that the plasmasphere, radiation belts, and ring current define overlapping regions in the dipole magnetosphere. We observe a trapped energetic electron population in the outer cusp on this orbit, generally during the two seasons per year when the POLAR orbit precesses through this region. Data from TIMAS and HYDRA on this day show that magnetosheath plasma was first encountered at 0100, at which time EFI showed an abrupt increase in broadband noise. HYDRA showed brief bursts of magnetosheath electrons between 0100-0230 that appeared to be anti-correlated with IES and HIST trapped energetic electrons. These short magnetosheath plasma encounters ceased by 0230 along with most of the EFI wave power.

In Figure 1 we plot time/energy/roll-angle spectrograms of phase space density from the CEPPAD/ HIST and CEPPAD/ IES electron instrument [Blake et al. (1995); Contos (1997)] on the POLAR spacecraft. The vertical stripes in the upper panels are an instrument artifact caused by mode switching of the HIST telescope. Successive panels are logarithmically spaced in energy where each panel displays the roll modulation (pitch angle) of the particles. The inset plots the count rates of selected energy bands linearly scaled from 0$20 \mathrm{cts} / \mathrm{s}$ as a function of pitch angle averaged over the interval 0100-0330 UT. The color scale displays the logarithm of $f\left(\mathrm{~s}^{3} / \mathrm{km}^{6}\right)$ from 0.00001 (purple) to 100 (red). From 0100-0315 UT the plot shows 30-1000 keV electrons with trapped pitch angle distributions located in the outer cusp at $L>10$. After $0330 \mathrm{UT}$ is an outer radiation belt traversal. Comparing the radiation belt and cusp loss cones, we see that the cusp's is much wider (FWHM $=90^{\circ}$ ), which is characteristic of a "leaky magnetic bottle". It also appears that the wide loss cone of the cusp is filled at a lower, isotropic level. Comparing the phase space densities at equal magnetic moment (black dots at a constant $7.4 \mathrm{keV} / \mathrm{nT}$ ), reveals that the outer cusp may have equal or higher phase space densities at $90^{\circ}$ than the outer radiation belts. However these two regions of high phase space density are bracketted in L-shell by regions of lower density: the polar cap UT $<0130$, the pseudo-trapping region of the outer zone, $0315<\mathrm{UT}<0345$, and the slot region separating the outer and inner zones at UT $>0500$. 


\section{Simulations}

Now this trapped cusp population is highly unusual because, classically speaking, the cusp cannot trap particles [Roederer(1970)]; it is not an "excluded region" in the Störmer theory of an ideal dipole [Störmer(1911); Rossi and Olbert(1970)]. However, the interaction of a magnetic dipole with the solar wind modifies the topology in a fundamental way; rather than a dipole, the cusp appears to be quadrupolar. We demonstrate the existence of this particle trap using the geomagnetic conditions of a nearly minimum latitude cusp and a nominal 11 Re standoff distance (Figure 2).

When we trace particles through this region we find trapping to occur when the electrons mirror around the local minimum of the field line found at the center of the cusp. The orbits take the shape of a lily, with a locally outward magnetic gradient instead of the typical inward gradient so that the particles drift $360^{\circ}$ around the cusp in an opposite sense to the trapped radiation belt particles. Our results show that 5-6000 keV electrons can

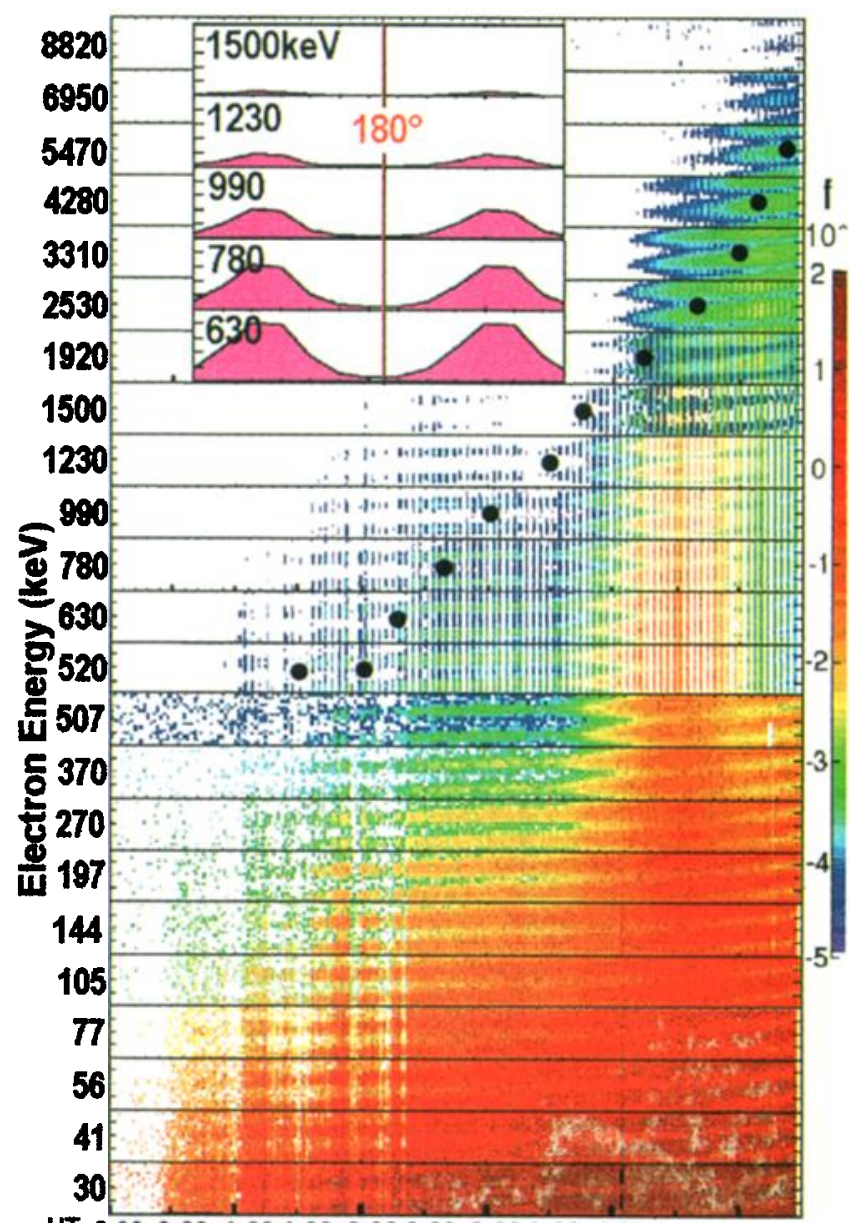

UT 0:00 0:30 1:00 1:30 2:00 2:30 3:00 3:30 4:00 4:30 5:00 5:30

L $36.828 .722 .7 \quad 18.214 .711 .9$ 9.66 7.82 6.305.05 4.07 3.50

Re 8.53 8.33 8.09 7.80 7.457.05 6.60 6.08 5.51 4.85 4.14 3.32

|B] 70.963 .573 .170 .370 .794 .9 128. 167. 220. 292. 451. 931 .

Figure 1. Electrons trapped in the cusp observed by POLAR/ CEPPAD. See text for details.
Table 1. Selected Periods of the Motion

\begin{tabular}{|c|c|c|c|c|c|c|}
\hline $\begin{array}{r}\mathrm{E} \\
\mathbf{k e V}\end{array}$ & $\begin{array}{r}\mu \\
k e V / n\end{array}$ & $\begin{array}{l}B_{0} \\
\text { nT }\end{array}$ & $\begin{array}{r}\alpha_{0} \\
\mathrm{deg}\end{array}$ & $\begin{array}{l}\tau_{0} \\
\sec \end{array}$ & $\begin{array}{l}\tau_{1} \\
\sec \end{array}$ & $\begin{array}{l}\tau_{2} \\
\text { sec }\end{array}$ \\
\hline 1000 & 32.2 & 26.4 & 41 & 0.004 & 1.0 & 77 \\
\hline 1000 & 30.7 & 21.2 & 35 & 0.006 & 1.1 & 67 \\
\hline 1000 & 43.4 & 12.8 & 32 & 0.009 & 0.6 & 28 \\
\hline 1000 & 294.9 & 6.7 & 88 & 0.016 & 0.1 & 1.3 \\
\hline 95 & 5.4 & 4.8 & 30 & 0.007 & 0.2 & 10 \\
\hline 5 & 4.5 & 1.1 & 85 & 0.040 & 0.4 & 14 \\
\hline
\end{tabular}

be trapped in the cusp of a T96 magnetosphere for $\tau>$ 300 seconds (i.e., many drift orbits), though admittedly without an electric field. Examination of particle trajectories in this region shows that although they lack a dipolar 2nd and 3rd invariant, since they never cross the dipole magnetic equator, we can find an analogous 2nd and 3rd "cusp" invariants of the motion if we define the "cusp equator" to be the surface of minimum $|B|$ along field lines that approach the cusp. Thus we can uniquely identify these invariants in analogy to a dipole by their pitch angle and $\left|B_{0}\right|$ at the crossing of the cusp equator. In Figure 2 one can see three nested "cuspshells" analogous to L-shells of the dipole. The limiting 2nd invariant of these trapped orbits occurs when the mirror point $\left|B_{m}\right|$ approaches the dayside equatorial field strength, at which point the electrons join the dipolar pseudo-trapped population and $\nabla B$-drift away from the cusp. From the pitch angle distribution, this value appears to be $\alpha_{0} \sim 60^{\circ}$. The spatial limit on the 3rd invariant is the maximum value of $\left|B_{0}\right|$ for which the "cusp equator" is still defined over a closed, $360^{\circ}$ loop.

Can these particles have come from the tail, that is, are they topologically connected to the nightside trapped particles that have drifted into the bifurcated dayside minimum? Yes, they are physically in the same region of space, but separated in phase space by very different 2nd invariants principally because the cusp $\left|B_{0}\right|$ minima are deeper than the corresponding tail minimum. Take for example a $50 \mathrm{keV} 90^{\circ}$ pitch-angle particle in the outer cusp, mirroring at $25 \mathrm{nT}$. For it to maintain the same magnetic moment while drifting, it must find a region of the magnetosphere with $\leq 25 \mathrm{nT}$ fields. The only other such region is deep in the tail, and topologically disconnected from the cusp, so that the particles remain trapped in the cusp and cannot drift away without destroying their first and second invariant. Conversely a $50 \mathrm{keV} 90^{\circ}$ pitch-angle particle trapped at midnight in a $50 \mathrm{nT}$ field can mirror through the cusp, but its pitch-angle when at the $25 \mathrm{nT}$ level must be $30^{\circ}$. Thus the faint background level inside the wide cusp loss cones could be understood as dipoletrapped particles, but if the first two invariants remain conserved, the peak at $90^{\circ}$ can only be locally trapped.

Why was this region not predicted previously? Just as Störmer theory, developed for an azimuthally symmetric dipole field, was recognized to predict trapping only after the discovery of the Van Allen belts, so 
this broken symmetry of a quadrupole cusp was recognized to predict trapping only after the observation of trapped cusp energetic particles [Chen et al.(1997)]. It is true that the existence of a cusp third invariant was speculated to partially explain the observation of $\mathbf{4 5 0}$ keV electrons [Antonova and Shabanskiy(1975); Shabansky(1971)], but these orbits were never calculated, and indeed, the observed trapped particles were identified with the 2nd invariant "branching" of a drifting dipole-trapped plasma. That is, the off-equatorial $\left|B_{0}\right|$ minimum and the resulting dayside bifurcation of drift orbits has been known since the Beard-Mead model [Mead and Beard(1964)], but the separate existence of a closed, non-bifurcated drift orbit around the cusp had not been previously demonstrated. Previous efforts at particle tracing in the cusp followed less energetic particles whose trajectories are dominated by electric field convection rather than $\nabla B$-drift, and therefore not trapped in the cusp [Delcourt et al.(1992)].

Recognizing that the cusp possesses three adiabatic invariants of the motion, we can characterize the trapped plasma in the cusp by direct analogy to the well-known dipole trap. High energy plasma is dominated by $\nabla B$ - drift whose closed drift paths form an annulus analogous to radiation belts. Low energy plasma is dominated by $E \times B$-drift, which because of the lack of an analogous corotation field, distorts the drift orbits and sweeps away plasma below some threshold energy, $\leq 30$ $\mathrm{keV}$. The finite size of the cusp produces energy- and mass-dependent inner and outer spatial limits on trapping, analogous to the inner and outer edge of the radiation belts. Finally, the limits on the strength of the mirror force arising from topological considerations produce analogous pitch angle loss cones. Unlike the dipole trap, in which the loss cone loses particles to atmospheric scattering and charge exchange, the cusp loss cone loses particles to the dipolar magnetosphere or the mantle, so in one sense, the cusp trap is half-embedded within the dipole trap.

All these analogies are true for a static magnetic cusp geometry, but are strongly modified by time variable fields, with occasions when $\Delta B / B \simeq 1$ [Chen et al.(1998)]. The dipole trap, by comparison, is extremely stable, with $\Delta B / B \ll 1$ over the majority of the trap volume. A second difference with far reaching effects is the separation of the periods of motion.

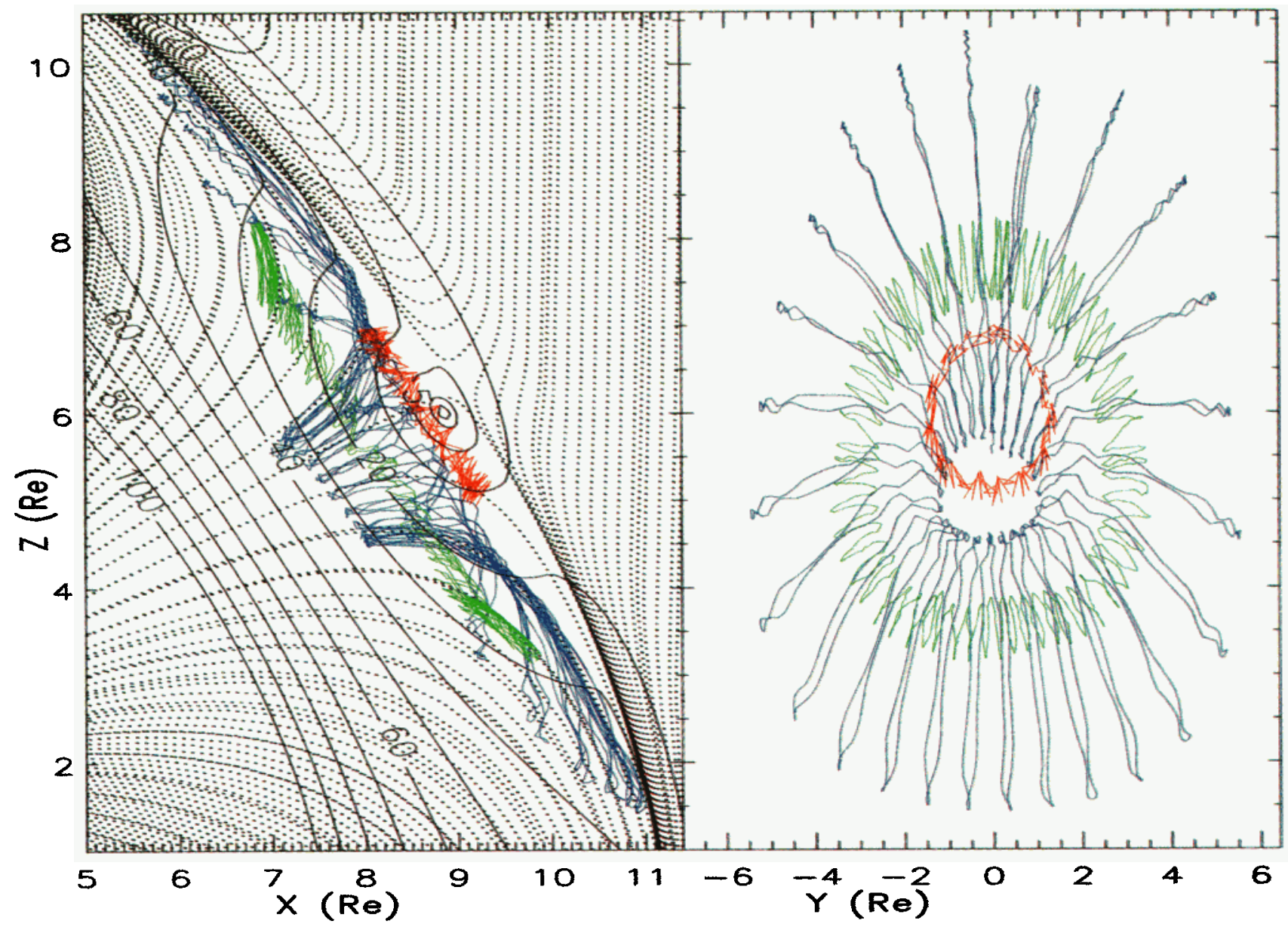

Figure 2. Trajectories of trapped $1 \mathrm{MeV}$ electrons in the the Earth's outer cusp, projected into the GSM X-Z and Y-Z planes. Dashed lines are field lines from the T96 magnetic field model (Dipole: June 21, 1996, 1300UT; Solar Wind: $+10 \mathrm{nT} \mathrm{Bz}, 1 / \mathrm{cm}^{3}$, and $\left.1000 \mathrm{~km} / \mathrm{s} \mathrm{V}_{S W}\right)$. Black lines are contours of $|B|$ in $\mathrm{nT}$. Green, blue and red trajectories correspond to the $1,3,4$ entries of Table 1 . 
The dipole trap has roughly three orders of magnitude separating the gyration, bounce and drift timescales [Lyons and Williams(1984)], whereas in the cusp trap these motions may be separated by less than an order of magnitude (See Table 1). Since the conservation of the adiabatic invariants depends upon the separation of timescales, the cusp trap is expected to be much more diffusive in energy and space than the dipole trap. This greater inherent diffusivity, coupled with the large perturbative power available would make the cusp an ideal location for resonance-broadened, chaotic acceleration [Arnol'd(1964)].

\section{Discussion and Conclusions}

We have shown that the POLAR spacecraft observed trapped $\mathrm{MeV}$ electrons in the Earth's cusp, and that these distributions are consistent with particles trapped in the outer cusp simulated using the Tsyganenko 96 model. Although this trapping geometry is quite different than the standard dipole geometry, we show that an analogous three invariants of the motion exist for this trapped population as well. The peak in the observed phase space density at the location of the outer cusp suggests both that the particles are being accelerated in situ and that they are a potential source population for diffusion into the dipole radiation belts. If so, this would provide a neat solution to the puzzle of the origin of the outer radiation belt electrons. However, diffusion in pitch-angle as well as in L-shell would be required to transport these particles from the outer cusp to the radiation belts, since the radiation belt pass is at a higher magnetic latitude which maps the trapped flux into the wide loss cones of the outer cusp. Furthermore, the minima between these trapping regions at 0330 UT indicates that any transport between them is either taking a circuitous route or is necessarily time-dependent. In a later paper we present the result of a time-dependent radial diffusion model of the outer radiation belt.

Acknowledgments. This analysis was supported by NASA contract NAS5-97147. We gratefully acknowledge the POLAR/ CEPPAD data provided by B. Blake and the POLAR/ MFE data provided by C. Russell.

\section{References}

Antonova, A. Ye. and V. P. Shabanskiy. Particles and the magnetic field in the outer noon magnetosphere of the earth. Geomag. and Aeron., 15, 243-247, 1975.

Amol'd, V. I. Dokl. Akad. Nauk. SSR, 156, 9, 1964.

Blake, J. B. et al. CEPPAD: Comprehensive energetic particle and pitch angle distribution experiment on POLAR. In C. T. Russell, editor, The Global Geospace Mission, pages 531-562. Kluwer Academic Publishers, 1995.

Chen, J., T. A. Fritz, R. B. Sheldon, H. E. Spence, W. N. Spjeldvik, J. F. Fennell, and S. Livi. A new temporarily confined population in the polar cap. Geophys. Res. Lett., 24, 1447-1450, 1997.

Chen, J., T. A. Fritz, R. B. Sheldon, H. E. Spence, W. N. Spjeldvik, J. F. Fennell, S. Livi, C. Russell, and D. Gurnett. Cusp energetic particle events: Implications for a major acceleration region of the magnetosphere. J. Geophys. Res., 109, 69-78, 1998.

Contos, A. R. Complete Description and Characterization of the High Sensitivity Telescope (HIST) Onboard the POLAR Satellite. Master's thesis, Boston University, Boston, MA, 1997.

Delcourt, D. C., T. E. Moore, J. A. Savaud, and C. R. Chappell. Nonadiabatic transport features in the outer cusp region. J. Geophys. Res., 97, 16,833-16,842, 1992.

Lyons, L. R. and D. J. Williams. Quantitative Aspects of Magnetospheric Physics. D. Reidel, Dordrecht, Holland, 1984.

Mead, G. D. and D. B. Beard. Shape of the geomagnetic field solar wind boundary. J. Geophys. Res., 69, 1181, 1964.

Roederer, J. G. Dynamics of Geomagnetically Trapped Radiation. Springer, New York, 1970.

Rossi, B. and S. Olbert. Introduction to the Physics of Space. McGraw-Hill Book Co., New York, 1970.

Shabansky, V. P. Some processes in the magnetosphere. Space Science Reviews, 12, 299-418, 1971.

Störmer, C. Sur les trajectories des corpuscules electrises dans l'espace sous l'actions des magnetisme terrestre avec application aux auarores boréales, seconde memoire. Arch. Sci. Phys. Nat. Ser. 4, 32, 117-123, 1911.

Tsyganenko, N. A. and D. P. Stern. Modeling the global magnetic field of the large-scale Birkeland current systems. J. Geophys. Res., 101, 27187-27198, 1996.

Van Allen, J. A., C. E. McIlwain, and G. H. Ludwig. Radiation observations with satellite 1958€. J. Geophys. Res., 64, 271-286, 1959.

BU Center for Space Physics, 725 Commonwealth Av, Boston, MA 02215 (e-mail: rsheldon@bu.edu, http: //buspace.bu.edu /EPG /rsheldon)

(Received April 2, 1998; accepted April 8, 1998.) 